\title{
A Collagen $\alpha 2(I)$ Mutation Impairs Healing after Experimental Myocardial Infarction
}

\author{
Ulrich Hofmann, ${ }^{*}$ Andreas Bonz, ${ }^{, \dagger}$ Stefan Frantz, ${ }^{*}$ \\ Kai Hu, ${ }^{*}$ Christiane Waller, ${ }^{\ddagger}$ Katrin Roemer, ${ }^{*}$ \\ Jürgen Wolf, ${ }^{*}$ Stefan Gattenlöhner, ${ }^{\S}$ \\ Johann Bauersachs, ${ }^{\text {" }}$ and Georg Ertl* \\ From the Comprehensive Heart Failure Center," University Clinic, \\ University of Würzburg, Würzburg; the Center for Cardiology, ${ }^{\dagger}$ \\ Lüneburg, Germany; the Clinic for Psychosomatic Medicine and \\ Psychotherapy, ${ }^{\ddagger}$ University Clinic, University of Ulm, Ulm; the \\ Center for Pathology, ${ }^{\S}$ Justus-Liebig University of Giessen, Giessen; \\ and the Hannover Medical School, "Tannover, Germany
}

Collagen breakdown and de novo synthesis are important processes during early wound healing after myocardial infarction (MI). We tested the hypothesis that collagen I, the main constituent of the extracellular matrix, affects wound healing after MI. The osteogenesis imperfecta mouse (OIM), lacking procollagen- $\alpha 2(I)$ expression, represents a model of the type III form of the disease in humans. Homozygous (OIM/ OIM), heterozygous (OIM/WT), and wild-type (WT/ WT) mice were subjected to a permanent myocardial infarction protocol or sham surgery. Baseline functional and geometrical parameters determined by echocardiography did not differ between genotypes. After MI but not after sham surgery, OIM/OIM animals exhibited significantly increased mortality, due to early ventricular rupture between day 3 and 7. Echocardiography at day 1 demonstrated increased left ventricular dilation in OIM/OIM animals. Less collagen I mRNA within the infarct area was found in OIM/OIM animals. At 2 days after MI, MMP-9 expression in the infarct border zone was higher in OIM/ OIM than in WT/WT animals. Increased granulocyte infiltration into the infarct border zone occurred in OIM/OIM animals. Neither granulocyte depletion nor MMP inhibition reduced mortality in OIM/OIM animals. In this murine model, deficiency of collagen I leads to a myocardial wound-healing defect. Both structural alterations within pre-existing collagen matrix and impaired collagen de novo expression contribute to a high rate of early myocardial rupture after MI. (Am J Pathol 2012, 180:113-122; DOI: 10.1016/j.ajpath.2011.09.033)
Osteogenesis imperfecta $(\mathrm{OI})$ is an inherited connective tissue disorder that occurs in 1 out of 20,000 to 1 out of 60,000 live births. ${ }^{1,2}$ The clinical phenotype is characterized by blue sclera, bone fractures, high incidence of hearing loss, joint laxity, restrictive pulmonary disease, and growth impairment. Patients with the milder types I and IV of osteogenesis imperfecta may have a normal life span and die of unrelated illnesses, such as myocardial infarction (MI) or malignancy. ${ }^{3}$ Human $\mathrm{OI}$ results from deficiencies in type I collagen procollagen- $\alpha 1$ or procollagen- $\alpha 2$ chains. The cardiac extracellular collagen matrix consists of more than $80 \%$ collagen I. ${ }^{4}$ Large coiled perimysial collagen fibers are major determinants of tensile stiffness of myocardial tissue. Ventricular rupture and wound-healing defects after cardiac surgery have been reported in patients with OI. ${ }^{5,6}$

Incidence of rupture of the left ventricular (LV) free wall as a complication of acute $\mathrm{Ml}$ is low in humans $(1 \%$ to $6 \%$ ), but mortality with this condition is very high $(60 \%$ to $90 \%){ }^{7,8}$ A recent retrospective analysis of autopsy records demonstrated that in $13 \%$ of cases of sudden death after $\mathrm{Ml}$ the underlying cause was ventricular rupture. ${ }^{9}$ Collagen degradation starts several hours after $\mathrm{Ml}$ and last for several days. ${ }^{10-12}$ The collagen matrix is degraded by activated serine proteases (eg, plasmin) and matrix metalloproteases (MMPs). Pro-MMP enzymes are stored extracellularly, bound to extracellular matrix (ECM) compounds, and are expressed by all cell types found in the myocardium, especially in response to inflammatory stimuli (for a detailed review, see Vanhoutte et $\mathrm{al}^{13}$ ). Importantly, besides transcriptional regulation, post-transcriptional activation and inhibition are subject to tight regulation within the extracellular space.

Supported by grants from the German Research Council (Deutsche Forschungsgemeinschaft) and SFB 688 (TPA10) (A.B., G.E., J.B., and S.F.).

Accepted for publication September 29, 2011.

U.H. and A.B. contributed equally to the present work.

Supplemental material for this article can be found at http://ajp. amjpathol.org or at doi: 10.1016/j.ajpath.2011.09.033.

Address reprint requests to Ulrich D.W. Hofmann, M.D., University of Würzburg, University Clinic, Comprehensive Heart Failure Center, Department of Internal Medicine I, Oberdürrbacherstrasse 6, D-97080 Würzburg, Germany. E-mail: hofmann_u2@klinik.uni-wuerzburg.de. 
Studies with genetically manipulated mice have demonstrated that MMP activation is a key factor for developing rupture after MI; targeted deletion of MMP-2 and MMP-9 in mice reduced the incidence of myocardial rupture. ${ }^{14-16}$ In the border zone of the infarct area, myofibroblasts start de novo synthesis of collagen between day 2 and day 3 after MI. ${ }^{11}$ Early collagen type III de novo synthesis is followed by collagen type I deposition, which contributes tensile strength to the infarcted tissue. ${ }^{17,18} \mathrm{~A}$ balance of ECM synthesis and degradation is required for maintaining tissue stability and preventing infarct expansion and rupture.

Because animal models have been developed in which $\mathrm{Ml}$ is induced by coronary ligature, we have gained much insight into inflammatory activation and ECM turnover during wound healing after MI. ${ }^{19,20}$ Osteogenesis imperfecta mice (OIM) harbor a recessively inherited guanine deletion on the Col1a2 gene, causing a complete loss of functional $\alpha 2(\mathrm{I})$ chains in homozygous mice (OIM/OIM). The OIM/OIM animals form homotrimeric type I collagen fibers, in which the $\alpha 2(I)$ chain is replaced by a third $\alpha 1(\mathrm{I})$ chain. In OIM/WT animals, both homotrimeric and heterotrimeric type I collagen fibers are found. ${ }^{21}$ OIM/OIM animals are born with fractures or develop them at an early stage. Joint subluxation, joint hemorrhage, and dorsal kyphosis are phenotypical correlates of a disorganized collagen matrix of the bone found in mice and in humans. Accordingly, both the thermal stability profile of homotrimeric type I collagen and the histological appearance of the bone in OIM/OIM animals is similar to those of patients with $\mathrm{OI} .{ }^{18} \mathrm{LV}$ collagen concentration is $45 \%$ lower than in wild-type animals, and collagen fiber diameter and number are decreased. LV chamber stiffness is reduced, despite increased collagen cross-linking in OIM/OIM. Heart weight/body weight ratio and basal LV function are similar across the OIM/OIM, WTMT, and OIM/WT genotypes. ${ }^{22}$

We investigated whether the genetic deficiency in collagen I in the OIM model affects wound healing and facilitates ventricular rupture after MI, assuming that the mechanism required depends on inflammatory cell infiltration and MMP activity.

\section{Materials and Methods}

\section{Animal Model}

We used male 12- to 18-week-old homozygous OIM/OIM $(n=51)$ and heterozygous OIM/WT mice $(n=39)^{18}$ and wild-type $(n=32)$ littermates, all weighing 22 to $32 \mathrm{~g}$. The cardiac phenotype of OIM has been reported previously. ${ }^{22}$ The OIM model is available from the Jackson Laboratory (no. 001815; Bar Harbor, ME) and was delivered by Charles River Laboratories International (Sulzfeld, Germany).

To allow acclimatization, animals were maintained for at least 2 weeks on a 12:12 hours light-dark cycle with free access to standard diet and water. A PCR method was used to determine mouse genotype, as described previously. ${ }^{23}$ The investigation conformed to the Guide for the Care and Use of Laboratory Animals published by the $\mathrm{NIH}$ (6th edition). All animal procedures were approved by the applicable local government agency.

\section{Infarct Model, Hemodynamic Measurement, and Study Design}

MI was induced in mice as described previously. ${ }^{24}$ In brief, anesthesia was induced by isoflurane inhalation. After intubation, mice were ventilated with a volume-cycled rodent respirator with air and isoflurane $(2 \%$ to $4 \%$, $\mathrm{v} / \mathrm{v}$ ) and were placed on a heating pad to preserve normothermia. After thoracotomy, ligation of the left coronary artery was performed with a 7-0 silk suture, 3 to $4 \mathrm{~mm}$ from the tip of the left auricle. Pallor, regional hypokinesia, and enlargement of the left ventricle confirmed the presence of an infarction. The chest wall was closed with a continuous 6-0 Prolene suture. In sham-operated animals, the suture was placed but not ligated. Buprenorphine was given routinely as analgesic during surgery. Antibiotics were not given during the procedure, but no apparent infection had developed by the time of necropsy. For analysis of MMP-9 and collagen I expression, animals were euthanized on day 2. Tissue was collected on day 2 after $\mathrm{Ml}$, to avoid loss of infarcted animals to spontaneous death. To investigate the effect of neutrophil depletion or metalloproteinase activity inhibition, animals were monitored for 8 days after permanent MI.

\section{Necropsy}

Mice were rigorously observed for morbidity and mortality after MI. Necropsy was performed after each death, to determine the cause of death. When blood in the pericardial sac or mediastinum indicated myocardial rupture, the whole left ventricle was carefully analyzed histologically, after embedding in paraffin and H\&E staining.

\section{Infarct Size}

On day 2, the left ventricle was rinsed with saline, opened longitudinally along the septum, and pressed flat between glass plates. For determination of infarct, size 8 mice per genotype were euthanized on day 2 . The left ventricle was then photographed and the area of infarction was quantified by planimetry (ImageJ software version 1.44; NIH, Bethesda, MD). Animals with small infarcts $(<30 \%$ LV area) were excluded from hemodynamic, echocardiographic, and molecular analyses.

\section{Echocardiography and Invasive Hemodynamic Assessment}

Echocardiography was performed on a Toshiba Aplio MX system with a $15-\mathrm{MHz}$ ultrasound probe, as described previously. ${ }^{24} \mathrm{~A}$ single researcher (U.H.) experienced in rodent echocardiography blinded to mouse genotype performed all echocardiographic analyses. Animals were serially imaged under light anesthesia with tribromoetha- 
nol/amylene hydrate (Avertin; 2.5\% w/v, $6 \mu \mathrm{L} / \mathrm{g}$ body weight i.p.) under spontaneous respiration. From twodimensional short-axis imaging, endocardial borders were traced at end-systole and end-diastole using an off-line analysis system (NICE; Toshiba Medical Systems Europe, Breda, The Netherlands). Measurements were performed at the apical and midpapillary muscle level. The end-systolic (smallest) and end-diastolic (largest) cavity areas were determined. Using these end-systolic and end-diastolic areas, fractional area changes were calculated as (end-diastolic area - end-systolic area)/ end-diastolic area. From two-dimensionally targeted Mmode tracings, end-diastolic diameter and end-systolic diameter were measured. Fractional shortening was calculated as (end-diastolic diameter - end-systolic diameter)/end-diastolic diameter. Only animals with an infarct size of $>30 \%$ and a heart rate of $>450$ beats/minute were included in the final analysis.

Hemodynamic measurements were performed according to published protocols under light isoflurane anesthesia (isoflurane $1.5 \%, \mathrm{v} / \mathrm{v}$ ). ${ }^{2} \mathrm{LV}$ pressure curves were recorded after catheter placement in the LV cavity; systolic and diastolic blood pressure measurements were obtained on catheter withdrawal in the thoracic aorta. LV performance was assessed by ejection fraction. LV preload was assessed by end-diastolic pressure (LVEDP, $\mathrm{mm} \mathrm{Hg}$ ), contractility by $\mathrm{d} P / \mathrm{d} t_{\max }(\mathrm{mm} \mathrm{Hg} /$ second) and $\left(\mathrm{d} P / \mathrm{d} t_{\text {max }}\right) / \mathrm{P}$, which is the peak rate of rise in ventricular pressure normalized to instantaneous developed pressure and relaxation by $-\mathrm{d} / \mathrm{d} t_{\max }(\mathrm{mm} \mathrm{Hg} /$ second).

\section{Immunohistochemistry}

Formalin-fixed sections of mouse myocardium were prepared according to standard procedures. Slides were labeled by sequential application of the primary polyclonal rat anti-mouse MAC-3 antibody (BD Pharmingen, Heidelberg, Germany), a primary rat anti-mouse neutrophil antibody (clone 7/4; Linaris, Wertheim, Germany), P4HB polyclonal antibody (Abcam, Cambridge, UK), or an irrelevant control antibody. Visualization was performed using a Vectastain avidin-biotin complex ABC kit, as indicated by the manufacturer (Vector Laboratories, Burlingame, CA). The slides were then washed, dehydrated, and mounted for light microscopy.

To analyze inflammatory cell infiltration, positively labeled cells were counted in high-power fields $(\times 400)$ by a blinded observer (technician S.K.). Semiquantitative grading for $\mathrm{P}_{4} \mathrm{HB}^{+}$cell infiltration was performed independently by two researchers (U.H. and technician S.K.) blinded for genotype on high-power fields $(\times 400)$. Grading was defined as follows: 0 indicates absence of positively stained cells in all fields examined; 1 indicates positive cells in at least one field; 2 and 3 indicate low and high density of positive cells, respectively, on more than one field; and 4 indicates a high density of positive cells in all fields examined.

\section{Analysis of Collagen Metabolism}

From infarct zones, procollagen- $\alpha 1(\mathrm{I})$ and procollagen$\alpha 2$ (I) mRNA was quantified by real-time PCR. ${ }^{24}$ After synthesis of CDNA with random hexamers (SuperScript; Invitrogen, Carlsbad, CA), real-time PCR was performed (iCycler system; Bio-Rad Laboratories, Hercules, CA) with commercially available TaqMan probes for 18S RNA, collagen type $1 \alpha_{1}$, and collagen type 1 $\alpha_{2}$ (Applied Biosystems, Foster City, CA). PCR parameters were used as recommended for the TaqMan universal PCR master mix kit (Applied Biosystems). RNA samples were normalized to $18 \mathrm{~S}$ rRNA.

Matrix metalloproteinase- 9 content in the infarct border zone was quantified with a commercial MMP-9 enzymelinked immunosorbent assay (R\&D Systems; Abingdon, UK) according to the manufacturer's protocol.

Picrosirius Red (PSR) polarization microscopy was performed to quantify interstitial collagen. Sections were analyzed from hearts of 5 OIM/OIM, 12 OIM/NT, and 4 WT/WT animals with Ml that survived the 56-day protocol. From each heart, one PSR-stained section derived from a midpapillary ring was photographed under polarized light with identical exposure times and was digitally analyzed according to a modified Junqueira method, as described previously. ${ }^{25}$ Gel zymography was performed as described previously. ${ }^{26}$ For assessment of the inhibitory effect of doxycycline, gels were incubated with $25 \mu \mathrm{g} / \mathrm{mL}$ doxycycline.

\section{Granulocyte Depletion and Metalloproteinase Inhibition}

Neutrophil granulocytes were depleted in 10 OIM/OIM animals by intraperitoneal administration of $250 \mu \mathrm{g}$ of an anti-Ly6G antibody (clone 1A8; BioLegend, Fell, Germany) ${ }^{27}$ either twice, during infarct surgery (day 0 ) and on day 2 , or once, on day 2. Animals were monitored for 7 days. For control of efficacy, $50 \mu \mathrm{L}$ of heparin-anticoagulated mouse blood was subjected to fluorescenceactivated cell sorting analysis on a FACSCalibur analyzer (BD Biosciences, San Jose, CA; Heidelberg, Germany) on day 2 in separate animals. Neutrophil granulocytes were identified within the leukocyte gate by direct staining of whole blood with biotinylated anti-CD45 and antiGR1-Cy5 antibodies (BD Biosciences). Before analysis, red blood cells were lysed by addition of $100 \mu \mathrm{L}$ of FixPerm buffer (eBioscience, San Diego, CA). Ten OIM/ OIM animals were treated intraperitoneally with doxycycline $30 \mathrm{mg} / \mathrm{kg}$ dissolved in $\mathrm{NaCl} 0.9 \%$ before surgery (day 0 ) and then orally $\left(100 \mathrm{mg} / \mathrm{kg}\right.$ ) on days 1 to $7^{28}$ Control animals were treated with $\mathrm{NaCl} 0.9 \%$.

\section{Statistical Analysis}

Results are presented as means \pm SEM per group. For single comparison of data, a $U$-test or, as appropriate, an unpaired $t$-test was performed. Two-way analysis of variance was performed for multiple comparisons. Survival data were analyzed by a log-rank test and visualized as Kaplan-Meier plots. Differences were consid- 
Table 1. Baseline Characteristics in OIM/OIM, WT/WT, and OIM/WT Mice

\begin{tabular}{cccc}
\hline & OIM/OIM & OIM/WT & WT/WT \\
\hline Sample size & $n=51$ & $n=39$ & $n=14$ \\
Body weight (g) & $24.2 \pm 0.6^{\star \dagger}$ & $28.7 \pm 0.5$ & $31 \pm 1.0$ \\
Heart rate & $469.0^{*} \pm 7.8$ & $462.6 \pm 7.7$ & $504.9 \pm 13.0$ \\
$\quad$ (bpm) & & & \\
Echo data, & & & \\
$\quad$ papillary & & & \\
ESA (cm $\left.{ }^{2}\right)$ & $4.5 \pm 0.3$ & $4.5 \pm 0.3$ & $3.8 \pm 0.5$ \\
EDA (cm $\left.{ }^{2}\right)$ & $9.0 \pm 0.3$ & $8.9 \pm 0.3$ & $8.7 \pm 0.4$ \\
LVDD (cm) & $0.34 \pm 0.01$ & $0.35 \pm 0.01$ & $0.33 \pm 0.01$ \\
LVSD (cm) & $0.24 \pm 0.01$ & $0.25 \pm 0.01$ & $0.23 \pm 0.01$ \\
Echo data, & & & \\
apical & & & \\
ESA (cm $\left.{ }^{2}\right)$ & $2.9 \pm 0.2$ & $3.1 \pm 0.2$ & $2.9 \pm 0.2$ \\
EDA (cm $\left.{ }^{2}\right)$ & $6.3 \pm 0.2$ & $6.6 \pm 0.2$ & $6.7 \pm 0.3$ \\
LVDD (cm) & $0.32 \pm 0.01$ & $0.32 \pm 0.01$ & $0.31 \pm 0.01$ \\
LVSD (cm) & $0.22 \pm 0.01$ & $0.25 \pm 0.01$ & $0.20 \pm 0.01$ \\
\hline
\end{tabular}

Data are expressed as means \pm SEM.

${ }^{*} P<0.05$, versus WT/WT

${ }^{+} P<0.05$, versus OIM $/$ WT

bpm; beats per minute; EDA, end-diastolic area; ESA, end-systolic area; LVDD, left ventricular diastolic diameter; LVSD, left ventricular systolic diameter

ered significant at $P<0.05$. Data analysis was performed using StatView software verison 3.0 (SAS Institute, Cary, NC) and WinSTAT version 2001.1 add-in to Microsoft Excel 2003 software (R. Fitch Software, Bad Krozingen, Germany).

\section{Results}

\section{Baseline Characteristics}

As determined by echocardiographic analysis, baseline LV geometry in OIM/OIM animals did not differ from that of WTMT or OIM/WT animals (Table 1). Body weight was significantly less for the OIM/OIM animals (Table 1).

\section{Survival and Cardiac Rupture after MI}

After $\mathrm{MI}$, there was a significant difference in mortality between OIM/OIM (65\%) and WT/WT (13\%) animals (Figure $1 \mathrm{~A})$. The most frequent cause of death during the first week after MI was LV rupture (Figure 1B). Ventricular rupture was identified in 13/22 OIM/OIM animals, compared with only 1/9 WT/WT animals. Histology typically revealed transmural rupture slits with white blood clots and intramural hemorrhage within the infarcted area (Figure 1D). The site of rupture was always confined to the infarct zone/border zone within the LV free wall. Incidence of rupture was independent of infarct size and was observed even in the absence of transmural infarction. In OIM/OIM animals subjected to sham surgery, mortality was significantly lower than in infarcted OIM/OIM animals, and no rupture was detected (Figure 1C).

\section{Left Ventricular Function and Geometry}

Echocardiography on day 1 demonstrated increased end-diastolic LV area (EDA) in OIM/OIM animals (OIM/ OIM $16.3 \pm 1.2 \mathrm{~mm}^{2}$; OIM/WT $10.3 \pm 0.5 \mathrm{~mm}^{2}$; WTMT $13.3 \pm 1.5 \mathrm{~mm}^{2} ; P<0.05$ ) (Figure 2, A and $\mathrm{C}$ ) and diameter (Figure 2, B and D) on apical short-axis sections reflecting the area of infarction. Infarct size did not differ among genotypes on day 2 (OIM/OIM $37.4 \pm 1.9 \%$; OIM/WT $38.4 \pm 1.7 \%$; WTMT $38.5 \pm 4.9 \%$ ). Invasive hemodynamic characterization on day 2 also did not reveal any significant difference (see Supplemental Table S1 at http://ajp.amjpathol.org).

\section{Collagen Metabolism}

Two days after $\mathrm{MI}$, there was significantly lower procollagen $\alpha 1(\mathrm{I})$ and $\alpha 2(\mathrm{I}) \mathrm{mRNA}$ expression in the infarct border zone in OIM/OIM animals. Expression of $\alpha 1(\mathrm{I})$, in arbitrary units, was $1.16 \pm 0.11$ for WT/WT and $0.45 \pm$
A

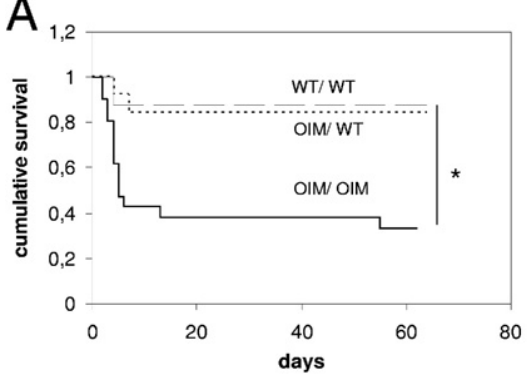

C

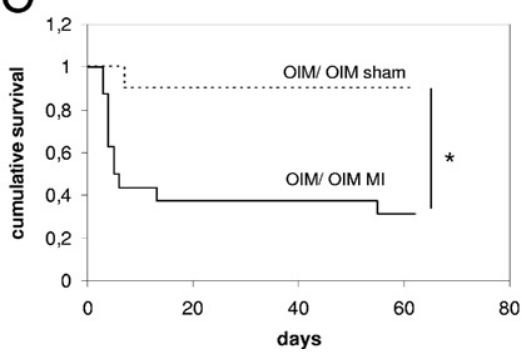

$\mathrm{B}_{5}$

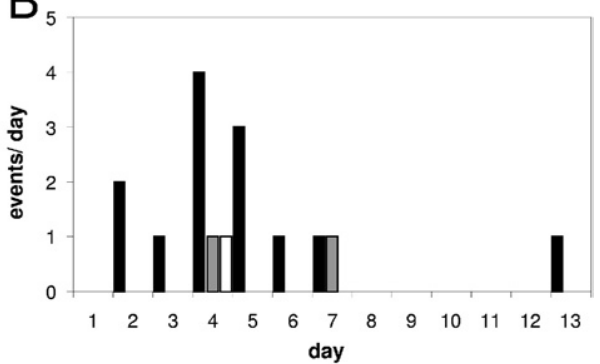

E

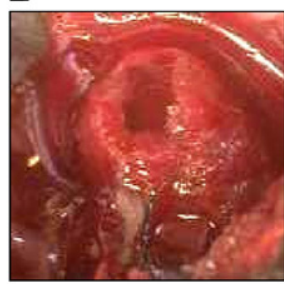

Figure 1. Survival after MI. A and C: Cumulative survival of the three genotypes after experimental MI. ${ }^{*} P<0.05$. B: Irrespective of genotype, rupture typically occurred during the first week after MI. Black bars, OIM/OIM $(n=22)$; gray, OIM/WT $(n=13)$; white, WT/WT $(n=9)$, Cumulative survival in infarct (MI) versus shamoperated OI mice. D: In OIM/OIM, necropsy often allowed macroscopic identification of large defects in ventricular free-wall. Ventricular rupture was always confirmed by histological analysis. Original magnification, $\times \sim 2$. E: A white blood clot (arrow) is apparent within a transmural rupture slit Original magnification, $\times 400$. 


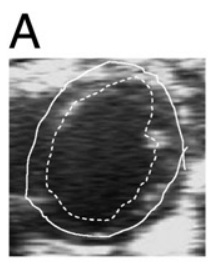

OIM/OIM

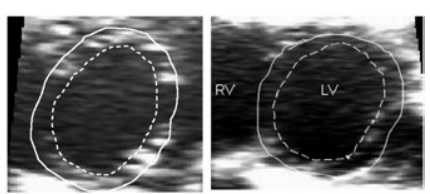

OIM/WT

WT/WT
B

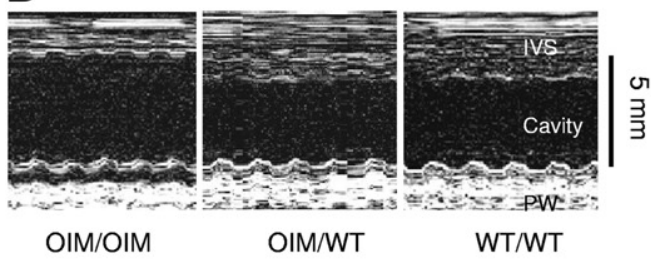

C

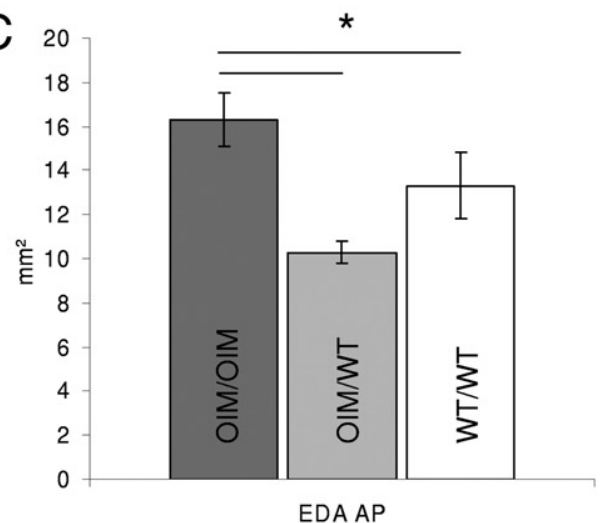

D

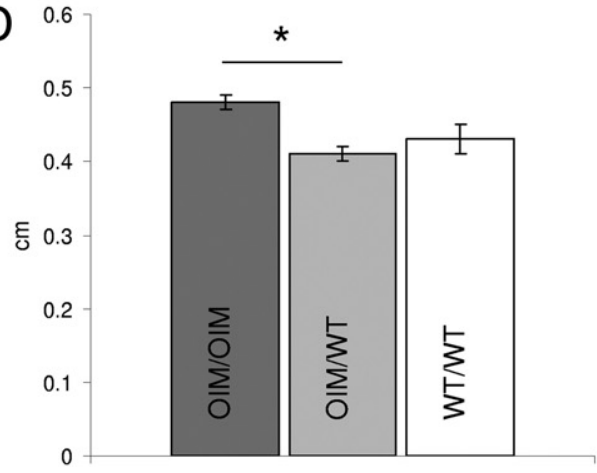

LVDD AP

Figure 2. Early LV dilation. Echocardiography on day 1 revealed greater LV dilation in OIM/OIM $(n=5)$ than in WT/WT $(n=4)$ animals after MI. A and C: End-diastolic area (EDA). B and D: LV end-diastolic diameter (LVDD). ${ }^{*} P<0.05$ versus OIM/OIM. AP, apical; IVS, interventricular septum; PW, posterior wall.

0.04 for OIM/OIM; expression of $\alpha 2(\mathrm{I})$ was $1.22 \pm 0.16$ for WTMT and $0.50 \pm 0.05$ for OIM/OIM $(P<0.05)$ (Figure $3 A)$. To determine whether this reduced collagen expression was due to lesser numbers of collagen-expressing cells, we analyzed the frequency of collagen-producing cells on day 2 by immunohistological staining for prolyl 4-hydroxylase (P4HB), an enzyme involved in hydroxylation of prolyl residues in pre-procollagen. The frequency of $\mathrm{P} 4 \mathrm{HB}^{+}$cells did not differ significantly between WT/WT and OIM/OIM animals on day 2 (Figure 3B). On day 7 , histology within the infarct zone of OIM/OIM animals in-

dicated only sparse collagen (Figure 4, D and E); furthermore, histology barely detected collagen in intact septal OIM/OIM myocardium (Figure 4F). These findings were confirmed by quantitative collagen analysis based on PSR staining (Figure 4, G-I).

Analysis of the mature scar 56 days after Ml demonstrated a modest but nonsignificant reduction in collagen fiber density (Figure 5E). The altered birefringent appearance of PSR-stained sections illustrates a qualitative difference in scar collagen composition (Figure 5, A-D), most likely because predominantly homotrimeric $\alpha 1(\mathrm{I})$ collagen was deposited in the myocardial scar, as is reported for healthy connective tissue in the OIM animal model. ${ }^{18}$
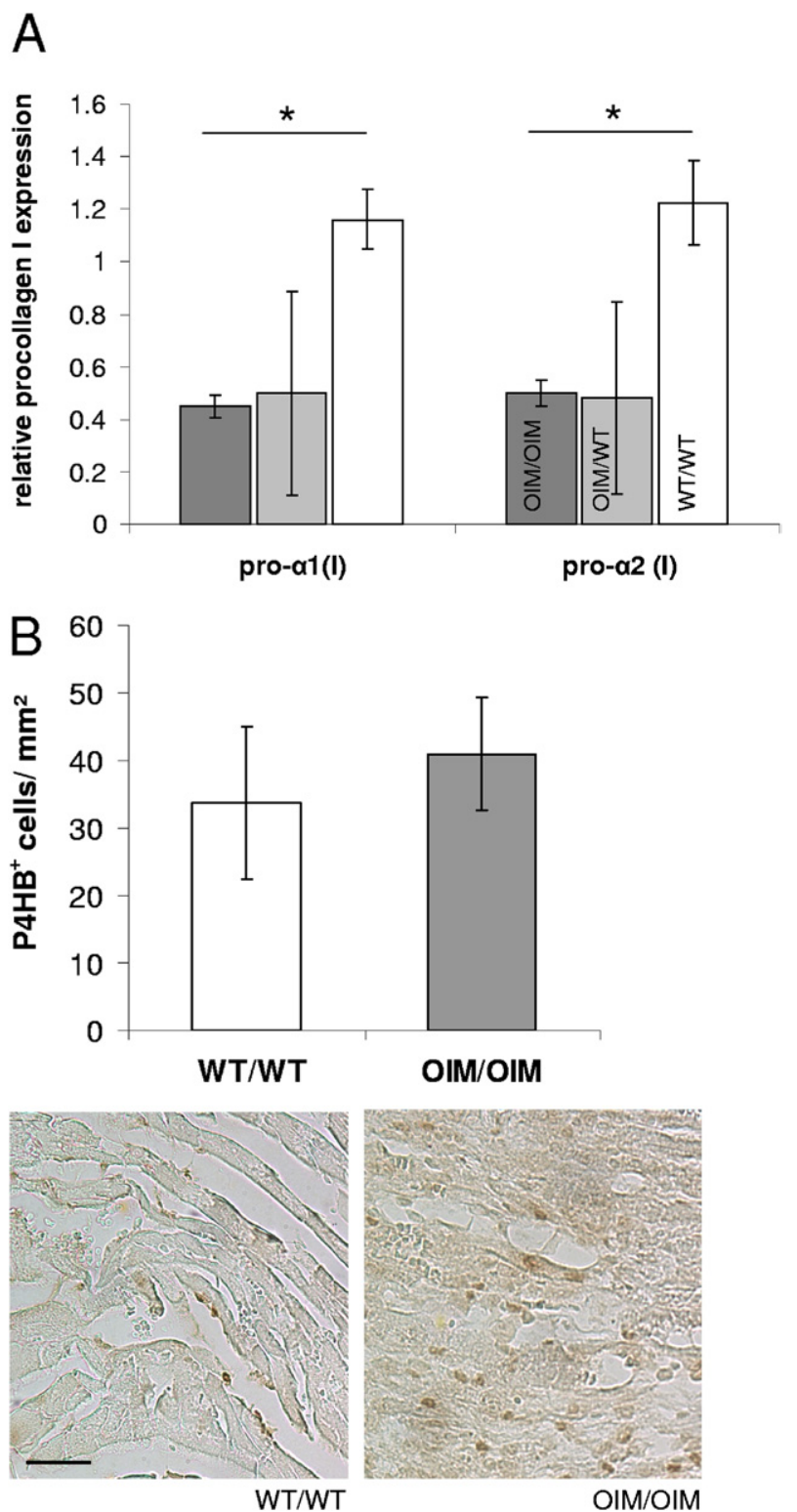

Figure 3. Collagen de novo expression after MI. A: Relative expression of procollagen $\alpha 1$ (I) and procollagen $\alpha 2$ (I) on day $2 .{ }^{*} P<0.05$, OIM/OIM versus WT/WT ( $n=3$ /group). B: Density of prolyl 4-hydroxylase-expressing $\left(\mathrm{P} 4 \mathrm{HB}^{+}\right)$cells within the infarct zone $(n=5 /$ group $)$. Scale bar $=10 \mu \mathrm{m}$. 

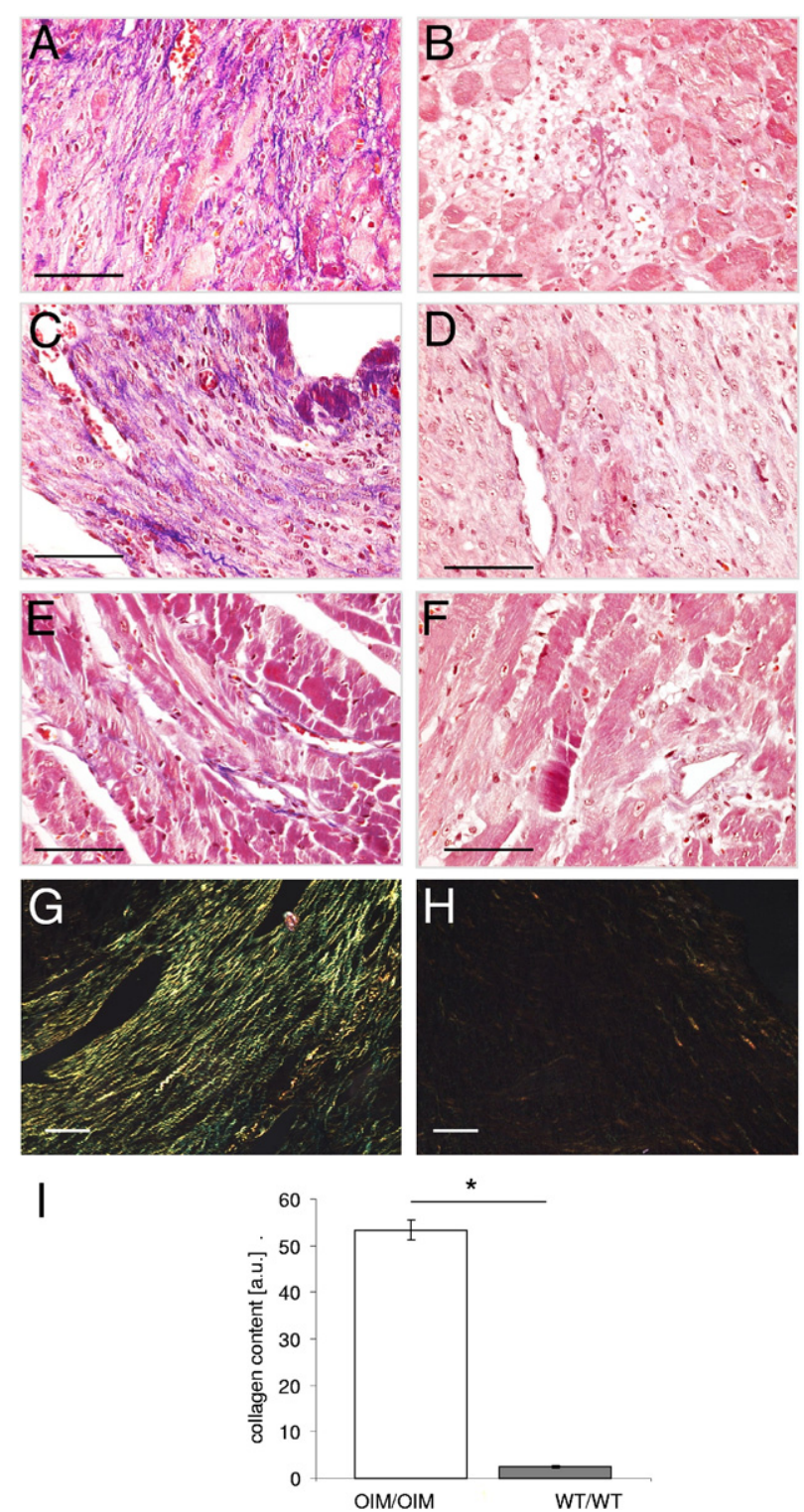

Figure 4. Extracellular collagen in WT/WT (left) and OIM/OIM (right) myocardium. On day 7 after myocardial infarction, Ladewig staining reveals any collagen fibers in blue within the infarcted myocardium (A-D) and within the intact septal myocardium $(\mathbf{E}$ and $\mathbf{F})$. Collagen fibers can be readily identified within the infarct border zone ( $\mathbf{A}$ and $\mathbf{B}$ ) and infarct zone ( $\mathbf{C}$ and D) in WT/WT animals (A, C and $\mathbf{E}$ ), but are barely detected in OIM/OIM animals $(\mathbf{B}, \mathbf{D}$, and $\mathbf{F})$. In septal myocardium, collagen can be found mainly in the perivascular space ( $\mathbf{E}$ and $\mathbf{F})$. $\mathbf{G}$ and $\mathbf{H}$ : PSR-stained sections. $\mathbf{I}$ : Quantitative analysis of collagen fiber area based on PSR-stained sections indicated only sparse collagen fibers within the infarct zone of OIM/OIM (H), compared with WT/WT $(\mathbf{G}) .{ }^{*} P<0.05$. a.u., arbitrary units. Scale bars: $10 \mu \mathrm{m}$.

Altogether, we found that both baseline interstitial collagen and de novo collagen formation are reduced in OIM/OIM animals, relative to WT/WT.

\section{Matrix Metalloproteinase-9 Expression}

In addition to collagen de novo expression, there is ongoing ECM degradation during early wound healing. At 2 days after MI, tissue levels of MMP-9 were significantly higher in OIM/OIM animals, both in the infarct border zone (OIM/OIM $4.47 \pm 0.71 \mathrm{ng} / \mu \mathrm{g}$, compared with WT/WT $2.38 \pm 0.74 \mathrm{ng} / \mu \mathrm{g} ; P<0.05)$ and in the remote, septal myocardium (OIM/OIM $2.80 \pm 1.90 \mathrm{ng} / \mu \mathrm{g}$, compared with WTMT $0.53 \pm 0.17 \mathrm{ng} / \mu \mathrm{g} ; P<0.05$ ) (Figure $6 \mathrm{~A}$ ). Because high MMP-9 activity has been associated with myocardial rupture in mice, we investigated whether MMP inhibition in OIM/OIM animals might reduce the mortality of OIM/OIM. Doxycycline acts as a broad-spectrum MMP inhibitor in vivo. We confirmed its activity in vitro against MMP-2 and MMP-9, the predominantly expressed MMPs in the infarcted myocardium (see Supplemental Figure S1 at http://ajp.amjpathol.org). Treatment with doxycycline for 7 days after Ml did not prevent excess mortality of OIM/OIM within the first week; there was $75 \%$ mortality in doxycycline-treated animals, compared with $50 \%$ mortality in control-treated OIM/OIM animals $(n=6$; $P>0.05)$. All animals died because of myocardial rupture.
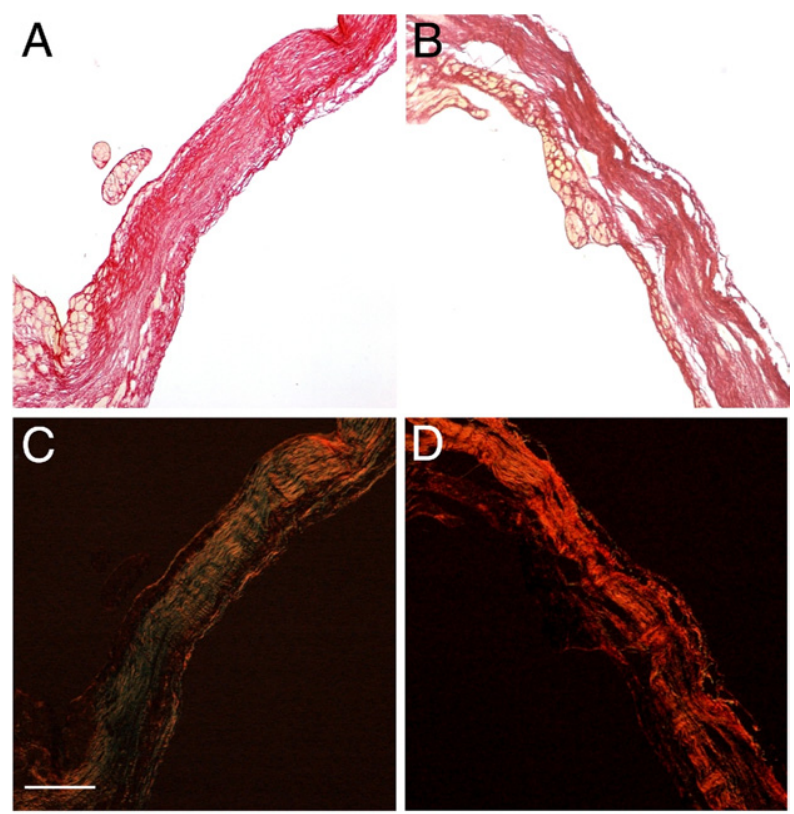

$\mathrm{E}$

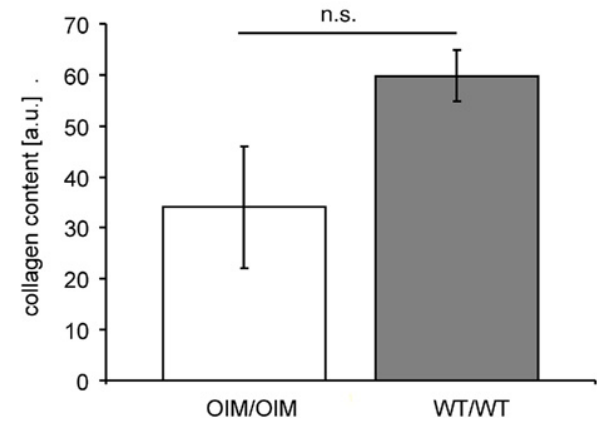

Figure 5. Analysis of collagen in the mature myocardial scar. At 56 days after MI, collagen within the infarct/infarct border zone was analyzed on PSRstained sections of OIM/OIM (A and $\mathbf{C}$ ) and WT/WT (B and D) animals. Analysis under polarized light (C and D) reveals the altered birefringent appearance of collagen fibers within the scar. E: Quantitative analysis of collagen fiber density indicated a nonsignificant reduction of total collagen content in OIM/OIM myocardial scar border zone $(P=0.14)$. a.u., arbitrary units. Scale bar $=100 \mu \mathrm{m}$. 

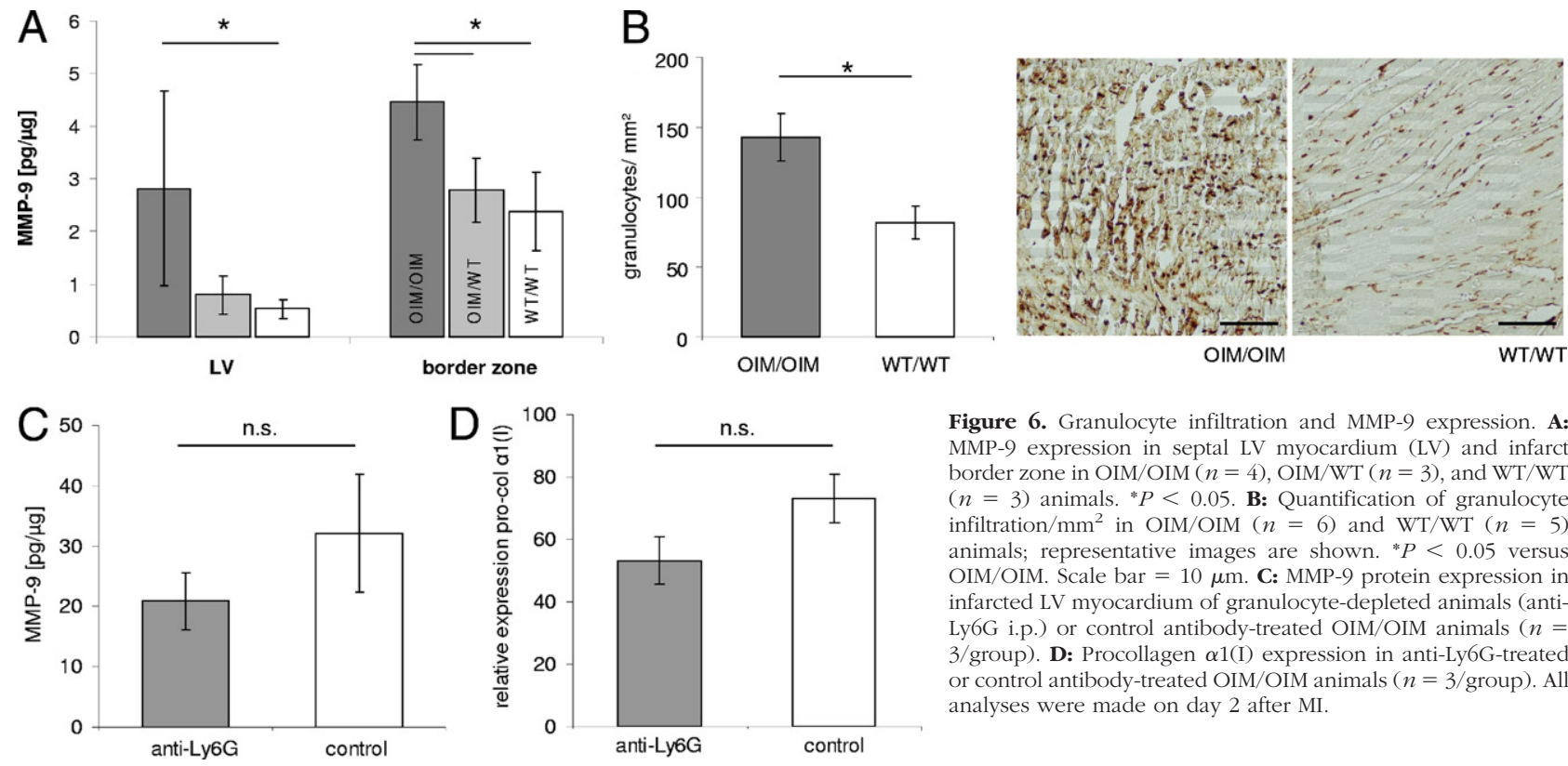

Figure 6. Granulocyte infiltration and MMP-9 expression. A MMP-9 expression in septal LV myocardium (LV) and infarct border zone in OIM/OIM $(n=4)$, OIM/WT $(n=3)$, and WT/WT $(n=3)$ animals. ${ }^{*} P<0.05$. B: Quantification of granulocyte infiltration $/ \mathrm{mm}^{2}$ in OIM/OIM $(n=6)$ and WT/WT $(n=5)$ animals; representative images are shown. ${ }^{*} P<0.05$ versus OIM/OIM. Scale bar $=10 \mu \mathrm{m}$. C: MMP-9 protein expression in infarcted LV myocardium of granulocyte-depleted animals (antiLy6G i.p.) or control antibody-treated OIM/OIM animals ( $n=$ $3 /$ group). D: Procollagen $\alpha 1$ (I) expression in anti-Ly6G-treated or control antibody-treated OIM/OIM animals ( $n=3$ /group). All analyses were made on day 2 after MI.

\section{Leukocyte Infiltration}

Granulocytes are the main leukocyte population and represent a pivotal source for MMPs during the first days after MI. Immunohistological analysis indicated increased granulocyte infiltration in the infarct border zone of OIM/OIM animals on day 2 (OIM/OIM $143 \pm 17 / \mathrm{mm}^{2}$, compared with WT/WT $82 \pm 12 / \mathrm{mm}^{2} ; P<0.05$ ) (Figure $6 \mathrm{~B})$ and only few macrophages (MAC-3 positive cells). There was no significant difference in macrophage infiltration among genotypes (data not shown).

To determine whether granulocytes were responsible for the observed phenotype, we depleted neutrophil granulocytes from peripheral blood with intraperitoneally injected anti-Ly6G antibodies. Anti-Ly6G treatment almost completely eliminated granulocytes from peripheral blood and largely prevented granulocyte infiltration in myocardium (see Supplemental Figure S2 at http:// ajp.amjpathol.org). However, granulocyte depletion increased the mortality from ventricular rupture in OIM/OIM animals during the first week after $\mathrm{Ml}(60 \%$ when antibody was administered on day 0 and day 2 and $50 \%$ when given at day 2 only, compared with $33 \%$ for the control; $n=10 /$ group; $P>0.05$ ). Granulocyte depletion had only a modest effect on MMP-9 expression within the infarcted myocardium, indicating that other infiltrating leukocytes or resident cardiac fibroblasts are an important source for MMP-9 (Figure 6C). Procollagen $\alpha 1(\mathrm{I})$ expression was also not significantly altered by granulocyte depletion (Figure 6D).

\section{Discussion}

The present findings demonstrate that mice with genetic deficiency of collagen type I are prone to fatal ventricular rupture within the first week after MI. Rupture occurred in a time frame consistent with previous reports for other murine strains after experimental MI. 29,30 Because rupture did not occur and mortality was low in sham-operated OIM/OIM animals, we attribute the high mortality in OIM/OIM animals to a specific susceptibility to myocardial injury. Thus, the OIM/OIM genotype serves as a model for deficient wound healing of MI. Importantly, in the present study the rupture rate was not related to infarct size, and there was no significant difference in infarct size on day 2 between genotypes. Therefore, wall thinning and dilation of a large infarct area was obviously not an indispensable prerequisite for fatal rupture.

Even OIM/OIM animals with nontransmural infarction developed rupture. This is a new and unexpected observation, fundamentally different from what has been previously reported for rupture pathology in mice ${ }^{31}$ and in humans. ${ }^{32}$ However, Lautsch and Lanks ${ }^{33}$ reported in a seminal article that intramural dissection often progresses in a stepwise manner through the myocardium before manifest rupture, which occurs when the dissection reaches the pericardial space. Therefore, the mechanical weakness of the OIM/OIM myocardium probably allows an intramural hematoma (which we often found in OIM/OIM animals; data not shown) to progress throughout the whole LV wall, even in the absence of an initial transmural injury.

The present findings are also in line with some clinical observations in patients with osteogenesis imperfecta. Valvular incompetence or aortic aneurysm requiring operative intervention is quite common. There are reports of perivalvular leakage and even ventricular rupture after cardiac surgery in these patients, indicating a particular defect of myocardial healing in the presence of collagen I deficiency or mutations. ${ }^{5,6}$ As previously pointed out, both the phenotypic and the molecular characteristics of the OIM/OIM strain closely duplicate those seen in patients with OI type $1 \mathrm{II} .{ }^{18}$ Weis et al ${ }^{22}$ reported significant structural and biomechanical alterations of ventricular 
myocardium in OIM/OIM animals ${ }^{22}$; however, LV function was not severely altered, indicating adaptation to the structural and biochemical alterations in the natural history of these animals. In accord with that report, we found that baseline geometry of OIM/OIM hearts did not differ from WT.

Various mechanisms have been identified in mouse models that promote ventricular rupture after MI. Several independent studies have demonstrated that inhibition or genetic deletion of MMPs protects against rupture. ${ }^{16,34,35}$ Proinflammatory cytokine expression promotes MMP activity and myocardial rupture. ${ }^{36}$ Deletion of class A macrophage scavenger receptor leads to increase in both MMP and proinflammatory cytokine expression, likely indicative of a prominent role of macrophages in the pathogenesis of myocardial rupture. ${ }^{37}$ In addition to excessive matrix breakdown, defective collagen I de novo formation (eg, in osteopontin-deficient mice) has also been reported to cause myocardial rupture. ${ }^{38}$ Furthermore, increased frequency of apoptosis in myocardium predisposes to rupture. ${ }^{39}$

The mechanism determining the occurrence of LV rupture in humans is less well characterized. From human hearts at autopsy, several types of rupture have been defined. ${ }^{40}$ Type I exhibits a clear-cut rupture with little intramural hematoma. Type II shows a multicanalicular myocardial dissection and extensive intramural bleeding. In type III, the orifice of rupture is covered either by a thrombus or by the pericardium. Type IV rupture is defined as incomplete or nontransmural. The type of rupture we observed resembles type II. In fact, in most OIM/OIM hearts, we found a diffuse interstitial deposition of erythrocytes, sometimes also forming a hematoma. Intramural hemorrhage is commonly seen in mice developing ventricular rupture, but the mechanism for local intramyocardial hemorrhage is unclear. ${ }^{31}$ Intramural bleeding contributes to accumulation of white blood cells, which are able to secrete matrix-degrading enzymes, and previous reports document more severe inflammatory cell accumulation in ruptured than in nonruptured human hearts. ${ }^{32,41}$ In accord with such reports, we found significantly enhanced granulocyte infiltration in the infarct border zones of OIM/OIM hearts on day 2. Because granulocytes are an important source for proteinase activity, increased granulocyte infiltration might have contributed to the high incidence of ventricular rupture. Granulocyte depletion only modestly reduced MMP-9 expression, indicating that other cells, especially monocytes/macrophages, contribute to local expression. However, granulocyte depletion did not prevent excess mortality in OIM/ OIM animals, arguing against an essential role of granulocytes for the observed phenotype.

A study characterizing mutant collagen I in OIM revealed that absence of the $\alpha 2(\mathrm{I})$ chain in collagen I results in an increased enzymatic susceptibility. ${ }^{21}$ Therefore, the homotrimeric collagen type I in OIM/OIM animals might be more susceptible to activated MMPs than is the wild-type collagen I. Furthermore, we found that MMP-9 expression on day 2 was significantly higher in OIM/OIM animals, compared with OIM/WT and WT/WT. A causal relationship between MMP-9 expression levels and myo- cardial rupture after MI was demonstrated by Heymans et al. ${ }^{14}$ In their mouse model, genetic deletion of MMP-9 protected against ventricular rupture after $\mathrm{MI}$, indicating a pivotal pathophysiological role for this enzyme in development of early myocardial rupture. We inhibited MMP activity by doxycycline, which attenuates myocardial MMP activity and prevents postinfarction remodeling in wild-type rats. ${ }^{42}$ In our model, doxycycline did not prevent rupture and mortality in OIM/OIM. Doxycycline is nonspecific, and it may interfere with other processes or may not have totally suppressed MMP activity. Furthermore, neutrophils are a source for various serine proteases facilitating ECM breakdown, and these are not inhibited by doxycycline.

Despite methodological limitations of the present experiments, previous findings in a mouse model with a cleavage-resistant collagen I mutation support our conclusion that enhanced breakdown of collagen I was probably not a main mechanism for dysfunctional wound healing in OIM/OIM animals. Lindsey et $\mathrm{al}^{43}$ reported that attenuated collagen I cleavage by MMPs did not affect post-MI wound healing.

Wound healing requires de novo expression of ECM proteins, such as collagen, which contribute to forming a mechanically stable scar. Procollagen III expression increases on day 2. whereas the expression of procollagen I, which is thought to bring mechanical strength to the myocardium, starts at day $4 .{ }^{44}$ The finding of increased rupture rate after day 3 in OIM/OIM animals coincides with the expression pattern of procollagen I, suggesting a causal relationship. However, expression of procollagen I in the infarct zone was lower in both OIM/OIM and OIM/WT animals than in WTMT animals. Thus, mechanisms other than or in addition to an insufficient early collagen-I de novo synthesis must contribute, because excessive rupture occurred in OIM/OIM but not in OIM/NT animals. In the present study, OIM/OIM myocardium had a lower baseline collagen content than either OIM/WT or WT/WT. Most likely, therefore, natively reduced myocardial strength due to the complete loss of functional $\alpha 2(\mathrm{I})$ collagen is the principal mechanism of rupture in OIM/OIM animals. However, we were not able to compare the biomechanical properties of the extracellular collagen network within the scar and intact myocardium in WT/WT and OIM/OIM animals. These have been characterized previously. ${ }^{22}$

In conclusion, a defect of the main myocardial ECM constituent collagen I does not severely affect baseline myocardial geometry and function, but does predispose to early rupture after myocardial infarction. The OIM model displays a myocardial wound-healing defect characterized by both a mechanical weakness of the preexisting ECM and insufficient de novo expression of stabilizing collagen I. Our findings are paralleled by clinical observations indicating that patients with $\mathrm{Ol}$ do not develop a specific cardiomyopathy, as could be expected from the prominent role of collagen I for myocardial ECM integrity, but do show disturbed myocardial wound healing after cardiac surgery. ${ }^{45}$ 


\section{Acknowledgments}

We thank Katharina Meder, Charlotte Dienesch, Susanne Knorr, and Helga Wagner for excellent technical assistance and Daniela Fraccarollo for her help with zymography.

\section{References}

1. Orioloi IM, Castilla EE, Barbosa-Neto JG: The birth prevalance rates for the skeletal dysplasias. J Med Genet 1986, 23:328-332

2. Martin E, Shapiro JR: Osteogenesis imperfecta: epidemiology and pathophysiology. Curr Osteoporos Rep 2007, 5:91-97

3. McAllion SJ, Paterson CR: Causes of death in osteogenesis imperfecta. J Clin Pathol 1996, 49:627-630

4. Robinson TF, Cohen-Gould L, Factor SM: Skeletal framework of mammalian heart muscle. Arrangement of inter- and pericellular connective tissue structures. Lab Invest 1983, 49:482-498

5. Lijoi A, Cisico S, Caputo E, Scarano F, Parodi E, Passerone GC: Left ventricular rupture after mitral valve replacement in a patient with osteogenesis imperfecta tarda. Tex Heart Inst J 1999, 26:295-297

6. Wong RS, Follis FM, Shively BK, Wernly JA: Osteogenesis imperfecta and cardiovascular diseases. Ann Thorac Surg 1995, 60:1439-1443

7. Reddy SG, Roberts WC: Frequency of rupture of the left ventricular free wall or ventricular septum among necropsy cases of fatal acute myocardial infarction since introduction of coronary care units. Am J Cardiol 1989, 63:906-911

8. McMullan MH, Maples MD, Kilgore TL Jr, Hindman SH: Surgical experience with left ventricular free wall rupture. Ann Thorac Surg 2001, 71:1894-1898; discussion 1898-1899

9. Pouleur AC, Barkoudah E, Uno H, Skali H, Finn PV, Zelenkofske SL, Belenkov YN, Mareev V, Velazquez EJ, Rouleau JL, Maggioni AP, Køber L, Califf RM, McMurray JJ, Pfeffer MA, Solomon SD; VALIANT Investigators: Pathogenesis of sudden unexpected death in a clinical trial of patients with myocardial infarction and left ventricular dysfunction, heart failure, or both. Circulation 2010, 122:597-602

10. Frantz S, Bauersachs J, Ertl G: Post-infarct remodelling: contribution of wound healing and inflammation. Cardiovasc Res 2009, 81:474481

11. Blankesteijn WM, Creemers E, Lutgens E, Cleutjens JP, Daemen MJ, Smits JF: Dynamics of cardiac wound healing following myocardial infarction: observations in genetically altered mice. Acta Physiol Scand 2001, 173:75-82

12. Tyagi SC, Campbell SE, Reddy HK, Tjahja E, Voelker DJ: Matrix metalloproteinase activity expression in infarcted, noninfarcted and dilated cardiomyopathic human hearts. Mol Cell Biochem 1996, 155: 13-21

13. Vanhoutte D, Schellings M, Pinto Y, Heymans S: Relevance of matrix metalloproteinases and their inhibitors after myocardial infarction: a temporal and spatial window. Cardiovasc Res 2006, 69:604-613

14. Heymans S, Luttun A, Nuyens D, Theilmeier G, Creemers E, Moons L, Dyspersin GD, Cleutjens JP, Shipley M, Angellilo A, Levi M, Nübe O, Baker A, Keshet E, Lupu F, Herbert JM, Smits JF, Shapiro SD, Baes M, Borgers M, Collen D, Daemen MJ, Carmeliet P: Inhibition of plasminogen activators or matrix metalloproteinases prevents cardiac rupture but impairs therapeutic angiogenesis and causes cardiac failure. Nat Med 1999, 5:1135-1142

15. Ducharme A, Frantz S, Aikawa M, Rabkin E, Lindsey M, Rohde LE, Schoen FJ, Kelly RA, Werb Z, Libby P, Lee RT: Targeted deletion of matrix metalloproteinase-9 attenuates left ventricular enlargement and collagen accumulation after experimental myocardial infarction. J Clin Invest 2000, 106:55-62

16. Hayashidani $S$, Tsutsui $H$, Ikeuchi M, Shiomi T, Matsusaka H, Kubota T, Imanaka-Yoshida K, Itoh T, Takeshita A: Targeted deletion of MMP-2 attenuates early LV rupture and late remodeling after experimental myocardial infarction. Am J Physiol Heart Circ Physiol 2003, 285:H1229-H1235

17. Cleutjens JP, Kandala JC, Guarda E, Guntaka RV, Weber KT: Regulation of collagen degradation in the rat myocardium after infarction. J Mol Cell Cardiol 1995, 27:1281-1292

18. Chipman SD, Sweet HO, McBride DJ JR, Davisson MT, Marks SC JR, Shuldiner AR, Wenstrup RJ, Rowe DW, Shapiro JR: Defective pro alpha $2(\mathrm{I})$ collagen synthesis in a recessive mutation in mice: a model of human osteogenesis imperfecta. Proc Natl Acad Sci USA 1993, 90:1701-1705

19. Ertl G, Frantz S: Wound model of myocardial infarction. J Am Physiol Heart Circ Physiol 2005, 288:H981-H983

20. Ertl G, Frantz S: Healing after myocardial infarction. Cardiovasc Res 2005, 66:22-32

21. McBride DJ JR, Choe V, Shapiro JR, Brodsky B: Altered collagen structure in mouse tail tendon lacking the alpha $2(\mathrm{I})$ chain. $\mathrm{J} \mathrm{Mol} \mathrm{Biol}$ 1997, 270:275-284

22. Weis SM, Emery JL, Becker KD, McBride DJ Jr, Omens JH, McCulloch AD: Myocardial mechanics and collagen structure in the osteogenesis imperfecta murine (oim). Circ Res 2000, 87:663-669

23. Saban J, King D: PCR genotyping of oim mutant mice. Biotechniques 1996, 21:190, -192

24. Frantz S, Hu K, Widder J, Bayer B, Witzel CC, Schmidt I, Galuppo P, Strotmann J, Ertl G, Bauersachs J: Peroxisome proliferator activatedreceptor agonism and left ventricular remodeling in mice with chronic myocardial infarction. Br J Pharmacol 2004, 141:9-14

25. Frantz S, Hu K, Bayer B, Gerondakis S, Strotmann J, Adamek A, Ertl G, Bauersachs J: Absence of NF-kappaB subunit p50 improves heart failure after myocardial infarction. FASEB J 2006, 20:1918-1920

26. Fraccarollo D, Galuppo P, Bauersachs J, Ertl G: Collagen accumulation after myocardial infarction: effects of ETA receptor blockade and implications for early remodeling. Cardiovasc Res 2002, 559 567

27. Daley JM, Thomas AA, Connolly MD, Reichner JS, Albina JE: Use of Ly6G-specific monoclonal antibody to deplete neutrophils in mice. J Leukoc Biol 2008, 83:64-70

28. Prall AK, Longo GM, Mayhan WG, Waltke EA, Fleckten B, Thompson RW, Baxter BT: Doxycycline in patients with abdominal aortic aneurysms and in mice: comparison of serum levels and effect on aneurysm growth in mice. J Vasc Surg 2002, 35:923-929

29. Cleutjens J, Creemers E: Integration of concepts: cardiac extracellular matrix remodeling after myocardial infarction. J Card Fail 2002, 8(6 Suppl):S344-S348

30. Gao XM, Dilley RJ, Samuel CS, Percy E, Fullerton MJ, Dart AM, Du XJ: Lower risk of postinfarct rupture in mouse heart overexpressing beta 2-adrenergic receptors: importance of collagen content. J Cardiovasc Pharmacol 2002, 40:632-640

31. Gao XM, Xu Q, Kiriazis H, Dart AM, Du XJ: Mouse model of postinfarct ventricular rupture: time course, strain- and gender-dependency, tensile strength, and histopathology. Cardiovasc Res 2005, 65:469-477

32. Oliva PB, Hammill SC, Edwards WD: Cardiac rupture, a clinically predictable complication of acute myocardial infarction: report of 70 cases with clinicopathologic correlations. J Am Coll Cardiol 1993, 22:720-726

33. Lautsch EV, Lanks KW: Pathogenesis of cardiac rupture. Arch Pathol 1967, 84:264-271

34. Krishnamurthy P, Peterson JT, Subramanian V, Singh M, Singh K: Inhibition of matrix metalloproteinases improves left ventricular function in mice lacking osteopontin after myocardial infarction. Mol Cell Biochem 2009, 322:53-62

35. Matsumura S, Iwanaga S, Mochizuki S, Okamoto H, Ogawa S, Okada Y: Targeted deletion or pharmacological inhibition of MMP-2 prevents cardiac rupture after myocardial infarction in mice. J Clin Invest 2005, 115:599-609

36. Sun M, Dawood F, Wen WH, Chen M, Dixon I, Kirshenbaum LA, Liu PP: Excessive tumor necrosis factor activation after infarction contributes to susceptibility of myocardial rupture and left ventricular dysfunction. Circulation 2004, 110:3221-3228

37. Tsujita K, Kaikita K, Hayasaki T, Honda T, Kobayashi H, Sakashita N, Suzuki H, Kodama T, Ogawa H, Takeya M: Targeted deletion of class A macrophage scavenger receptor increases the risk of cardiac rupture after experimental myocardial infarction. Circulation 2007, 115:1904-1911

38. Trueblood NA, Xie Z, Communal C, Sam F, Ngoy S, Liaw L, Jenkins AW, Wang J, Sawyer DB, Bing OH, Apstein CS, Colucci WS, Singh K: Exaggerated left ventricular dilation and reduced collagen deposition after myocardial infarction in mice lacking osteopontin. Circ Res 2001, 88:1080-1087

39. Matsusaka H, Ide T, Matsushima S, Ikeuchi M, Kubota T, Sunagawa $\mathrm{K}$, Kinugawa S, Tsutsui $\mathrm{H}$ : Targeted deletion of $\mathrm{p} 53$ prevents cardiac 
rupture after myocardial infarction in mice. Cardiovasc Res 2006 70:457-465

40. Sutherland FW, Guell FJ, Pathi VL, Naik SK: Postinfarction ventricular free wall rupture: strategies for diagnosis and treatment. Ann Thorac Surg 1996, 61:1281-1285

41. Atkinson JB, Robinowitz M, McAllister HA, Virmani R: Association of eosinophils with cardiac rupture. Hum Pathol 1985, 16:562-568

42. Villarreal FJ, Griffin M, Omens J, Dillmann W, Nguyen J, Covell J: Early Short-Term Treatment With Doxycylin Modulates Postinfarction Left Ventricular Remodeling. Circulation 2003, 108:1487-1492

43. Lindsey ML, Yoshioka J, MacGillivray C, Muangman S, Gannon J, Verghese A, Aikawa M, Libby P, Krane SM, Lee RT: Effect of a cleavage-resistant collagen mutation on left ventricular remodeling [Erratum appeared in Circ Res 2004, 95: e34]. Circ Res 2003, 93:238-245

44. Cleutjens JP, Verluyten MJ, Smiths JF, Daemen MJ: Collagen remodeling after myocardial infarction in the rat heart. Am J Pathol 1995, 147:325-338

45. Migliaccio S, Barbaro G, Fornari R, Di Lorenzo G, Celli M, Lubrano C, Falcone S, Fabbrini E, Greco E, Zambrano A, Brama M, Prossomarit G, Marzano S, Marini M, Conti F, D'Eufemia P, Spera G: Impairment of diastolic function in adult patients affected by osteogenesis imperfecta clinically asymptomatic for cardiac disease: casuality or causality? Int J Cardiol 2009, 131:200-203 